\title{
Enhanced Monitoring of Antiretroviral Resistance in Persons Living with HIV/AIDS
}

\author{
James T. Gomez*, Shuang Wang, Syed Rizvi and Salma K. Khuwaja \\ Houston Health Department, Bureau of Epidemiology, Houston, TX, USA
}

\section{Objective}

The aim of this presentation is to illustrate a public health surveillance method for monitoring antiretroviral drug resistance (ADR) in persons diagnosed with human immunodeficiency virus infection (HIV). We developed procedures for examining HIV related electronic laboratory reports (ELR) transmitted to our health department surveillance system that link to corresponding clinical and demographic data for patients with varying degrees of antiretroviral drug resistance.

\section{Introduction}

The implementation of the Health Information Technology for Economic and Clinical Health (HITECH) Act of 2009, permits health departments with the authority to establish programs to improve health care quality by the promotion of health IT and includes guidelines for receiving and transmitting secure electronic health information. When ELR health data has been verified for completeness, it enhances the ability to monitor HIV diagnosed patients for virologic failure that in some cases is associated with ADR. In recent years the transmission of HIV drug-resistant strains among persons with HIV has been an on-going concern. When drug-resistant HIV becomes resistance to more than one drug class, the control of viral replication becomes more difficult. Consequently, measuring the burden of antiretroviral resistance has risen in importance, ranking alongside other major public health concerns when planning HIV prevention campaigns. The analytical method illustrated here aims to improve monitoring of patients with increased viral replication that may lead to poor clinical outcomes and resistance to antiretroviral medications, leading to significant increases in monetary costs when prescriptions for secondline drug regiments are required [1].

\section{Methods}

The automation of data collection was optimized by uploading ELRs into the Houston Department and Human Services Health (HDHHS) disease surveillance system, a collaborative effort involving health facilities and information technology (IT) staff for establishing connectivity using national standards (HL7 format, LOINC and SNOMED coding). A laboratory event package for HIV was customized for populating drug resistance data for different classes of antiretrovirals (e.g., Nucleoside and Nucleotide RT Inhibitors (NNRTI), NonNucleoside RT Inhibitors (NRTI), Protease Inhibitors (PI), etc.) that includes CD4 and viral load counts. An automated query for accessing this information was developed for linking this file to demographic and clinical data to examine records that exceeded viral load thresholds and resistance across different classes of drugs. In an initial analysis, we selected ten months of laboratory resistance data results ( 857 records) reported by ELR to HDHHS from 1-2013 to 10-2013 on adults receiving HIV outpatient care from clinics affiliated with a large local public hospital system.

\section{Results}

A total of 294 patients (34\%) were shown to have resistance or possible resistance to at least one class of antiretrovirals. Of those with resistance, $113(38 \%)$ had resistance in two or more classes of drugs and $8(3 \%)$ patients were found to have resistance to three classes. Resistance was most common for NNRTI's 215 (73\%), followed by NRTI's 112 (38\%), and PI's 54 (18\%). A higher proportion of males (36\%) than females (34\%) had resistance to one or more drug classes $(\mathrm{RR}=1.04 ; \mathrm{CI}=85-1.27)$. Antiretroviral resistance was weakly linked to substance abuse within one year of resistance testing $(\mathrm{RR}=$ 1.07; $\mathrm{CI}=90-1.25)$.

\section{Conclusions}

HHDHS developed a method for evaluating HIV resistance at the community level. We intend to provide health partners in the community with information to monitor groups in which resistance to antiretrovirals has been detected, especially in those with resistance to multiple drug classes to improve their health outcome. Further refinements in surveillance analytical methods are planned to correlate resistance data to other clinical and laboratory data for developing a comprehensive profile of this group.

\section{Keywords}

Anti-retroviral drug resistance; Electronic data collection; HIV

\section{Acknowledgments}

1 - Staff of the HIV/STD Surveillance Program, HDHHS Bureau of Epidemiology

2 - Jilin Province, China branch of the Center for Disease Control and Prevention

3 - This work was supported by cooperative agreements from the Centers for Disease Control and Prevention (CDC). Its contents are solely the responsibility of the authors and do not necessarily represent the official views of the Centers for Disease Control and Prevention.

\section{References}

Smith, RD; Coast, J; (2012) The economic burden of antimicrobial resistance: Why it is more serious than current studies suggest. Technical Report. London School of Hygiene \& Tropical Medicine, London.

\footnotetext{
*James T. Gomez

E-mail: james.gomez@houstontx.gov
} 